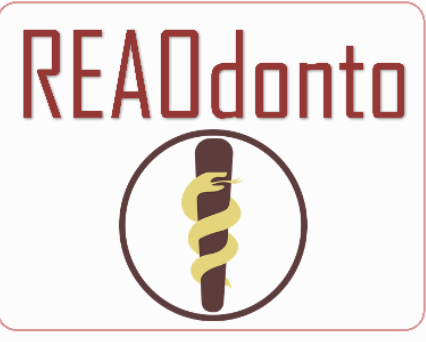

Revista Eletrônica Acervo Odontológico

\section{ESTUDO DE CASO}

Recebido em: 11/2020

Aceito em: $12 / 2020$

Publicado em: $1 / 2021$

\title{
Manifestações clínicas bucais da paracoccidioidomicose: um relato de caso
}

\author{
Oral clinical manifestations of paracoccidioidomycosis: a case report
}

\section{Manifestaciones clínicas orales de paracoccidioidomicosis: reporte de un caso}

Caroline Maria de Souza ${ }^{1}$, Fernanda Barbosa Esteves Rocha ${ }^{1}$, Mirella Moreira Miguel ${ }^{1}$, Sarah Vitor Teixeira Rodrigues ${ }^{1}$, Roseli Teixeira Miranda ${ }^{1}$, Maria Carolina Vaz Goulart ${ }^{1}$, Gérsika Bitencourt Santos ${ }^{1 *}$.

\begin{abstract}
Resumo: A Paracoccidioidomicose é uma doença fúngica decorrente do fungo dimórfico Paracoccidioides brasiliensis que possui predileção por lugares quentes e úmidos, por essa razão há uma grande incidência da doença no Brasil. O sítio primário da doença envolve a região pulmonar, mas com o avanço dos estudos voltados à doença, a região da mucosa bucal vem sendo bastante afetada. $O$ artigo tem como objetivo demonstrar as manifestações clínicas bucais da paracoccidioidomicose em uma paciente do sexo feminino diagnosticada aos 79 anos, diagnosticada pela equipe de odontologia e encaminhada para clínica médica para tratamento da doença. A paciente apresentou lesão ulcerada de fundo moriforme com pontos hemorrágicos no rebordo alveolar direito. Foi realizada a biópsia incisional e o resultado microscópico revelou estruturas birrefringentes sugestiva de Paracoccidioidomicose. A paciente foi diagnosticada pela equipe de odontologia da clínica odontológica de uma universidade particular e posteriormente encaminhada ao setor de Infectologia de um hospital universitário de Alfenas-MG para avaliação e tratamento dos sintomas sistêmicos. Embasado nos resultados dos exames físico, clínico e histológicos e nas características que predispõe à susceptibilidade a doença, como ser tabagista há mais de 30 anos, hipertensa e trabalhadora da zona rural, levou-se ao diagnóstico final da lesão.
\end{abstract}

Palavras-chave: Paracoccidioidomicose, Manifestações orais, Fungo.

\begin{abstract}
Paracoccidioidomycosis is a fungal disease resulting from the dimorphic fungus Paracoccidioides brasiliensis that has a predilection for hot and humid places, which is why there is a high incidence of the disease in Brazil. The primary site of the disease involves the pulmonary region, but with the advance of research and studies focused on the disease, the region of the oral mucosa has been greatly affected by the fungus. The article aims to demonstrate the oral clinical manifestations of paracoccidioidomycosis in a female patient who was diagnosed at 79 years old, treated with oral antifungal by the dental team and referred to a medical clinic for treatment of the disease. The patient had an ulcerated lesion with a moriform background with hemorrhagic stitches on the right alveolar ridge. The incisional biopsy was performed and the microscopic result revealed birefringent structures suggestive of Paracoccidioidomycosis. The patient was diagnosed by the dental team at the dental clinic of a private university and later referred to the infectious diseases department of a university hospital in Alfenas-MG for the evaluation and treatment of systemic symptoms. Based on the results of the physical, clinical and histological exams and on the
\end{abstract}

1 Universidade José do Rosário Vellano (UNIFENAS). Alfenas - MG. *E-mail: gersika.santos@unifenas.br 
characteristics that predispose to susceptibility to the disease, such as being a smoker for more than 30 years, hypertensive and working in the countryside, led to the final diagnosis of the injury.

Keywords: Paracoccidioidomycosis, Oral manifestations, Fungus.

Resumen: La paracoccidioidomicosis es una enfermedad fúngica resultante del hongo dimórfico Paracoccidioides brasiliensis que tiene predilección por los lugares cálidos y húmedos, razón por la cual existe una alta incidencia de la enfermedad en Brasil. El sitio primario de la enfermedad involucra la región pulmonar, pero con el avance de las investigaciones y estudios enfocados en la enfermedad, la región de la mucosa oral se ha visto muy afectada por el hongo. El artículo tiene como objetivo demostrar las manifestaciones clínicas bucales de la paracoccidioidomicosis en una paciente de sexo femenino que fue diagnosticada a los 79 años, tratada con antifúngico oral por el equipo odontológico y remitida a una clínica médica para el tratamiento de la enfermedad. El paciente presentaba una lesión ulcerada de fondo moriforme con suturas hemorrágicas en el reborde alveolar derecho. Se realizó biopsia incisional y el resultado microscópico reveló estructuras birrefringentes sugestivas de Paracoccidioidomicosis. El paciente fue diagnosticado por el equipo odontológico de la clínica odontológica de una universidad privada y posteriormente derivado al departamento de enfermedades infecciosas de un hospital universitario de Alfenas-MG para la evaluación y tratamiento de síntomas sistémicos. A partir de los resultados de los exámenes físicos, clínicos e histológicos y de las características que predisponen a la susceptibilidad a la enfermedad, como ser fumador durante más de 30 años, hipertenso y trabajar en el campo, llevaron al diagnóstico definitivo de la lesión.

Palabras clave: Paracoccidioidomicosis, Manifestaciones orales, Hongos.

\section{INTRODUÇÃO}

A Paracoccidioidomicose (PCM) é uma doença fúngica decorrente do fungo dimórfico Paracoccidioides brasiliensis que possui predileção por lugares quentes e úmidos, por essa razão há uma grande incidência na América Latina, principalmente no Brasil, em regiões Sul, Sudeste e Centro-Oeste. O fungo se dissemina pelo pulmão podendo atingir os demais órgãos através da corrente sanguínea e linfática, causando um comprometimento sistêmico (MOREIRA APV, 2008).

A PCM é infecção micótica sistêmica, profunda e granulomatosa, a Paracoccidioidomicose ou Blastomicose Sul Americana pode ser adquirida por via inalatória, por lesões na pele ou nas mucosas, com maior frequência no gênero masculino, raça de pele branca, faixa etária entre 41 e 50 anos e principalmente aqueles que exercem alguma função na atividade rural (NORONHA FILHO OL, VALENTE C, 2011).

A infecção é resultante da inalação desses esporos do fungo, que se alastram chegando até a cavidade oral, caracterizando sua manifestação em lábios, língua, faringe e mucosa jugal. Aparecem como lesões ulceradas, infiltradas e duras à palpação com numerosos pontos hemorrágicos. De modo geral a doença é semelhante ao quadro de pneumonia aguda, incluindo febre e falta de ar e agravando para perda de peso, dificuldade de deglutir e insônia (WANKE B, AIDÊ MA, 2009).

Perante a suspeita dessa infecção o indicado é a realização de uma anamnese dirigida exemplificando os sinais e sintomas, juntamente com as informações detalhadas pelo paciente e assim encaminhá-lo para a verificação dessa suspeita realizando uma radiografia de tórax, hemograma completo, provas da função hepática, ureia, creatinina, sódio, potássio e exame sorológico para PCM e a biópsia tecidual (incisional) (YASUDA MA, et al., 2017). O diagnóstico de PCM é baseado na detecção de elementos fúngicos de P. brasiliensis por exame histopatológico de espécimes corados com hematoxilina-eosina. A coloração com ácido periódico-schiff (PAS) e Grocott-Gomori também são técnicas de coloração úteis (ARRUDA JAA, et al., 2018).

O tratamento é através do uso de medicamentos antifúngicos com administração por via oral, sendo utilizado com maior frequência o Cetoconazol, Fluconazol e Itraconazol, em casos mais graves devem ser 
tratados com sulfametoxazol/trimetoprim. A duração do tratamento varia de cada paciente, dependendo da severidade da doença (NEVILLE BW, et al., 2016). A incidência das lesões bucais da PCM requer atenção nas políticas públicas de saúde para diagnóstico e tratamento (ARRUDA JAA, et al., 2018).

$\mathrm{Na}$ cavidade bucal se apresenta como estomatite moriforme, de evolução lenta, com fundo de aspecto de finas granulações vermelhas e pode estar sob a forma de ulceração, com dores, sangramento, mobilidade dos dentes e sialorreia (BISINELLI JC, et al., 2001). Sendo assim, o presente estudo relata um caso clínico de PCM, destacando a importância do cirurgião-dentista na conduta clínica multiprofissional adequada.

\section{DETALHAMENTO DO CASO}

Paciente, 79 anos, gênero feminino, leucoderma, trabalhadora rural, residente em Machado-MG. Foi encaminhada para a Clínica Odontológica de um hospital universitário de Alfenas-MG em novembro de 2009, com história de lesão ulcerada de fundo moriforme com pontos hemorrágicos, no rebordo alveolar direito, medindo cerca de dois centímetros em seu maior diâmetro. Apresentando sintomatologia dolorosa, no rebordo alveolar e ao engolir, também apresentou linfonodos submandibulares infartados do tipo inflamatório. Apresentava também outras lesões em semi mucosa e face interna de lábio.

A evolução foi de aproximadamente um ano. À época, a paciente referiu ser tabagista e hipertensa, características que predispõe a susceptibilidade à doença. Além disso confirmou que trabalhou na zona rural durante sua vida adulta, sendo esta sua principal atividade laboral.

Foram solicitados ao Laboratório de Análises Clínicas os seguintes exames: hemograma, glicemia em jejum, sorologia para paracoccidioidomicose e radiografia PA de tórax. As hipóteses diagnósticas foram de Paracoccidioidomicose e Carcinoma epidermóide, por isso foi feita a biopsia incisional como exame complementar. No exame macroscópico viam-se dois fragmentos de tecido mole, consistência elástica, forma irregular, superfície irregular, cor castanho-enegrecido, medindo $25 \times 5 \times 3 \mathrm{~mm}$.

Microscopicamente, os cortes corados em HE mostram fragmento de mucosa bucal constituído por epitélio pavimentoso, estratificado, paraqueratinizado, hiperplásico, irregular, com crescimento pseudoepiteliomatoso, com áreas de exocitos e focos de abscesso. Subjacente, na lâmina própria, nota-se o tecido conjuntivo fibroso denso com intenso infiltrado inflamatório mononuclear e polimononuclear, com vasos sanguíneos dilatados e hiperêmicos, com áreas de microedema. Foram notadas algumas células gigantes multinucleares e estruturas birrefringentes sugestivas de Paracoccidioides brasiliensis. Confirmando assim, o resultado histológico de paracoccidioidomicose. Foi dado o encaminhamento para o setor de Infectologia do Hospital para avaliação e tratamento do caso.

\section{DISCUSSÃO}

Paracoccidioidomicose é uma infecção fúngica profunda causada pelo fungo dimórfico Paracoccidioides brasiliensis. É uma doença rara no mundo, mas endêmica, com a maior prevalência na América do Sul e Brasil, particularmente (TRINDADE AH, et al., 2017).

Os primeiros indícios do aparecimento da doença paracoccidioidomicose foram em abril de 1908, no Brasil, onde dois pacientes chegaram ao hospital Santa Casa em São Paulo queixando-se de lesões na cavidade bucal. Desse modo procederam-se exames a fim de identificar o fator causal, descobrindo através dos estudos histológicos um novo parasita, um fungo. Com o passar dos anos outros casos foram surgindo e outros cientistas foram aprimorando ainda mais as pesquisas iniciais, com isso foi descoberto além do fungo Paracoccidioides brasiliensis, também o fungo Paracoccidioides lutzi, concluindo então que existem duas espécies diferentes de fungos causadores de tal doença (MOREIRA APV, 2008).

Em um levantamento brasileiro, a PCM oral representou $0,3 \%$ dos casos entre as lesões orais e maxilofaciais diagnosticadas. A disponibilidade de informações sobre a manifestação oral da PCM ainda é escassa. Estudos da Argentina e do México, no entanto, não forneceram a frequência relativa de lesões biopsiadas. De acordo o estudo de Arruda JAA, et al. 2017, a maioria dos casos era de países em 
desenvolvimento em áreas com umidade elevada do solo. Dos 613 casos de PCM oral, apenas 10 eram pacientes de países desenvolvidos; no entanto, eles tinham um histórico de viagens para alguma área endêmica.

Na América Latina, principalmente no Brasil é relatado o maior índice de endemia da doença. É uma doença fúngica que vai desde sintomas leves à sintomas severos, podendo ser de caráter sistêmico atingindo os principais órgãos do corpo humano e levando ao óbito. A explicação sobre tal doença é que o fungo tem predileção por lugares quentes e úmidos, o solo é um lugar apropriado para sua disseminação, por isso os esporos produzidos pelo fungo infectam o solo e matéria vegetal em decomposição, desse modo a infecção no ser humano ocorre devido a inalação desses esporos seguida de lesão pulmonar primária e posterior disseminação linfática ou hematogênica (FORTES MRP, et al., 2011).

O estudo de Fornajeiro $\mathrm{N}$, et al. (2005) realizou um inquérito epidemiológico sobre PCM na região noroeste do Paraná por meio de intradermorreação com gp43 e avaliou algumas condições epidemiológicas que reforçam a importância da PCM na região. A positividade foi de $43 \%$, o que, aliado às condições ambientais favoráveis ao desenvolvimento do fungo, reforçam que esta região seja endêmica para PCM e também reservárea de $P$. brasiliensis, confirmando os dados já conhecidos de que as regiões Sul, Sudeste e Centro-Oeste do Brasil são as mais acometidas pela micose (TOLENTINO ES, et al., 2010).

O solo é a principal fonte natural de conídios infecciosos, e as pessoas que vivem em áreas rurais representam um grupo de risco para PCM. Recentemente, um surto de PCM foi relatado em uma região brasileira, onde ocorreu o desmatamento e a remoção massiva de terra. De acordo com o período de incubação e as características dos indivíduos afetados, existem duas formas clínicas de PCM, que é aguda/subaguda e crônica. A forma crônica (tipo adulta) responde por cerca de $80 \%$ dos casos de PCM, principalmente entre os trabalhadores rurais do sexo masculino. A forma aguda / subaguda (tipo juvenil) costuma afetar indivíduos de até 30 anos de ambos os sexos (ARRUDA JAA, et al., 2018).

Os principais fatores de risco para infecção por PCM são atividades ocupacionais relacionadas ao manejo do solo contaminado por $P$. brasiliensis, como agricultura e jardinagem. Neste caso clínico a paciente é produtora rural, o que aumentou o risco de infecção. A evolução para uma lesão clinicamente detectável é consequência de um desequilíbrio entre a relação hospedeiro e parasita que contribui para uma resposta imune prejudicada que favorece a multiplicação fúngica e a disseminação sistêmica (PELLICIOLI ACA, et al., 2015).

A manifestação clínica mais frequente da PCM crônica é a prevalência pelo gênero masculino, pacientes imunossuprimidos, alcoolistas e fumantes sendo os que possuem maior susceptibilidade à doença, atingindo pessoas entre 10 e 20 anos de idade, entretendo pode acontecer de os sintomas só aparecerem entre dos 30 aos 60 anos, devido à baixa imunidade. Na maioria das vezes a infecção fúngica vai ser assintomática e o sistema imunológico vai tentar destruir o parasita, ocorrendo formação de granulomas que limitam a disseminação do fungo. Quando ocorre a baixa imunidade o paciente pode apresentar sintomas de pneumonia, febre, sudorese, tosse e falta de ar, além das manifestações bucais.

De acordo com Trindade AH, et al. 2017, em geral, a PCM é relatada em pacientes com idades entre 2102 anos, mas a prevalência é maior entre 30 e 60 anos e menos frequente durante a primeira década de vida e após os 70 anos. A paracoccidioidomicose prevalece entre os homens, mas a proporção masculino: feminino varia de acordo com a região geográfica. No Sudeste e Centro-Oeste do Brasil, varia de 3: 1 a 10: 1, atingindo proporções maiores, perto de 100: 1 em número de casos do sul do Brasil. Neste caso clínico a paciente apresenta 79 anos de idade, é do sexo feminino e reside na região sudeste do país. Nesse caso relatado a paciente de 79 anos, relatou durante a anamnese que era tabagista há mais de 30 anos e trabalhava na zona rural, apresentou lesão na gengiva localizado no rebordo inferior e não apresentava nenhum outro sintoma a não ser a dor ao engolir, nesse caso a paciente não apresentava dificuldades para respirar (YASUDA MA, MARTINEZ R, 2017). É uma doença que pode ser fatal e que se manifesta com um amplo espectro de apresentações clínicas, incluindo frequentes lesões. As manifestações da mucosa oral frequentemente representam o principal sinal clínico representando números representativos entre 39,38\% e $50 \%$ dos pacientes (TRINDADE AH, et al., 2017). 
A confirmação diagnóstica dessa micose deve ser feita por métodos laboratorial. O melhor e mais rápido meio de estabelecer o diagnóstico desta doença fúngica se faz pelo exame direto de espécimes clínicas, o que permitirá a visualização dos elementos fúngicos. $O$ exame direto a fresco, entre lâmina e lamínula, em meio a uma gota de hidróxido de potássio, tem um excelente rendimento". No referido caso adotou a realização de biópsia incisional como diagnóstico, levando em conta a maior veracidade do resultado possibilitando um diagnóstico mais preciso e completo. Foi solicitado exames como hemograma, glicemia em jejum, sorologia para paracoccidioidomicose e radiografia PA de tórax, que posteriormente foram avaliados pela área médica (TOLENTINO ES, et al., 2010).

O caso clínico apresenta a importância da atuação do cirurgião-dentista no tratamento de um paciente com infecção sistêmica. Destaca-se que a paracoccidioidomicose pode apresentar sequelas graves ou evoluir ao óbito, caso o diagnóstico seja retardado ou o tratamento mal conduzido. É importante que o profissional esteja atento para as manifestações bucais e seja capaz de estabelecer o diagnóstico correto desta enfermidade. Considerando que o objetivo deste trabalho foi expor especificamente as manifestações bucais da paracoccidioidomicose, revela-se a importância do cirurgião-dentista no reconhecimento das lesões e diagnóstico desta infecção sistêmica, sendo de extrema importância a abordagem desses pacientes no início, visando um tratamento adequado para que o paciente consiga uma melhor qualidade de vida e um prognóstico favorável (SILVA MACN, et al., 2015).

A PCM trata-se de uma doença fúngica e apresenta alto índice endêmico no Brasil, portanto nos revela importância de estarmos atentos a todos os sintoma e sinais que o paciente apresentar, pois a mucosa bucal é um sítio de desenvolvimento da doença. A experiência adquirida com a observação das lesões bucais desta paciente confirma a importância do conhecimento do cirurgião dentista ao realizar um diagnóstico, tratamento e prognóstico precoce, deste modo diminuindo o índice de mortalidade de tal doença, além de instruir os pacientes mais susceptíveis a terem hábitos positivos, como uso adequado dos equipamentos de proteção individuais padronizados aos trabalhadores rurais e evitar o consumo de tabaco e álcool.

\section{REFERÊNCIAS}

1. ARRUDA JAA, et al. A multicentre study of oral paracoccidioidomycosis: Analysis of 320 cases and literature review. Oral Diseases. 2018; 24 (8): 1492-1502.

2. BISINELLI JC, et al. Manifestações Estomatológicas da paracoccidioidomicose. Revista Brasileira de Otorrinolaringologia, 2001; 67(5), 683-687.

3. FORNAJEIRO N, et al. Paracoccidioidomycosis epidemiological survey using gp43, in two cities of northwestern region of Paraná, Brazil., 2005; 38(2): 191-3.

4. FORTES MRP, et al. Imunologia da paracoccidioidomicose. An. Bras. Dermatol. 2011; 86(3), 516-524.

5. MOREIRA APV. Paracoccidioidomicose: histórico, etiologia, epidemiologia, patogênese, formas clínicas, diagnóstico laboratorial e antígenos. BEPA. Boletim Epidemiológico Paulista (Online), 5(51), 11-24.

6. NEVILLE BW, et al, Patologia Oral \& Maxilofacial, 4ํed, Rio de Janeiro, Ed. Elsevier, 2016;1810 p.

7. NORONHA FILHO OL, VALENTE C. Paracoccidioidomicose com manifestação bucal - Casuística em Volta Redonda-RJ nos anos de 2005 a 2008. Rev Univ Vale do Rio Verde, 2011; 9(2):24-34.

8. PELLICIOLI ACA et al. Synchronous Oral Paracoccidioidomycosis and Pulmonary Tuberculosis in an Immunocompetent Patient. Mycopathologia, 2015; 179(5-6):459-464.

9. ROCHA GJNM, et.al. Paracoccidioidomicose palpebral: Relato de três casos. Arq. Bras. Oftalmol, 2002; 65(5): 575-578.

10. SILVA MACN, et al. Paracoccidioidomicose pulmonar com manifestações orais: relato de caso. NewsLab 2015; (127):66-8.

11. TOLENTINO ES, et al. Manifestações da paracoccidioidomicose - considerações gerais e relato de caso. RFO; 2010, 15(1):71-76.

12. TRINDADE AH, et al. Oral paracoccidioidomycosis: Retrospective analysis of 55 Brazilian patients. Mycoses, 2017; 60:521-525.

13. WANKE B, AIDÊ MA. Paracoccidioidomicose - capítulo 6. Jornal Brasileiro de Pneumatologia, 2009; 35(12):12451249.

14. YASUDA MA, et al. Il consenso brasileiro em paracoccidioidomicose-2017. Epidemiologia e Serviços de Saúde, 27, e0500001. 